\title{
Regulatory interplay between soybean root and soybean cyst nematode during a resistant and susceptible reaction
}

\author{
Parsa Hosseini ${ }^{1,2,3^{*}}$ and Benjamin F Matthews ${ }^{3}$
}

\begin{abstract}
Background: Plant-parasitic nematodes (PPNs) are obligate parasites that feed on the roots of living host plants. Often, these nematodes can lay hundreds of eggs, each capable of surviving without a host for as long as 12 years. When it comes to wreaking havoc on agricultural yield, few nematodes can compare to the soybean cyst nematode (SCN). Quantifying soybean (Glycine max) transcription factor binding sites (TFBSs) during a late-stage SCN resistant and susceptible reaction can shed light onto the systematic interplay between host and pathogen, thereby elucidating underlying cis-regulatory mechanisms.

Results: We sequenced the soybean root transcriptome at 6 and 8 days upon independent inoculation with a virulent and avirulent SCN population. Genes such as $\beta-1,4$ glucanase, chalcone synthase, superoxide dismutase and various heat shock proteins (HSPs) exhibited reaction-specific expression profiles. Several likely defense-response genes candidates were also identified which are believed to confer SCN resistance. To explore magnitude of TFBS representation during SCN pathogenesis, a multivariate statistical software identified 46 over-represented TFBSs which capture soybean regulatory dynamics across both reactions.

Conclusions: Our results reveal a set of soybean TFBSs which are over-represented solely throughout a resistant and susceptible SCN reaction. This set furthers our understanding of soybean cis-regulatory dynamics by providing reaction-specific levels of over-representation at 6 and 8 days after inoculation (dai) with SCN.
\end{abstract}

Keywords: Soybean, Soybean cyst nematode, SCN, Transcription factor binding site

\section{Background}

Obligate parasites, such as plant-parasitic nematodes (PPNs), are infamously known for their ability to suppress host defense mechanisms and cripple yield of many agricultural crops. Such devastation is tightly orchestrated by nematode effector proteins that commandeer hostplant metabolic machinery. One of the most destructive PPNs to soybean yield is the soybean cyst nematode (SCN; Heterodera glycines). Worldwide, approximately 1.5 billion dollars in soybean yield is lost annually due to SCN infestations [1,2]. In SCN susceptible soybeans, this devastation begins when the female juvenile-stage 2 (J2)

\footnotetext{
*Correspondence: parsa.hosseini@nih.gov

1 School of Systems Biology, George Mason University, Manassas, VA, USA

${ }^{2}$ Computational Biology Branch, National Center for Biotechnology

Information, National Institutes of Health, Bethesda, MD, USA

Full list of author information is available at the end of the article
}

nematode penetrates the host root. J2 effector proteins are injected into the root, dissolving plant cell walls and driving formation of a metabolically-active, multinucleated feeding site known as a syncytium [3]. Newly-molted $\mathrm{J} 3$ males and females feed from this nutrient-rich syncytium, subsequently molt into J4 larvae and copulate [4]. After approximately 30 days post-copulation, a hardened sac of SCN eggs known as a cyst becomes visible to the naked-eye. In the resistant reaction however, cysts are not visible since $\mathrm{J} 2$ nematodes can neither form a nutrientrich syncytium nor copulate. Thus, J2 nematodes starve to death.

With next-generation sequencing (NGS) now becoming a central assay in transcriptomics, entire transcriptomes can now be sequenced at unprecedented resolution. Fueled by the economic impact of SCN infestations, 
numerous studies have utilized NGS assays to sequence and quantify the soybean transcriptome [5-8].

In this study, we extend such works by conducting transcriptomic and regulatory analyses on soybean roots (Peking cv.) inoculated with $\mathrm{SCN}$. We sequence the soybean root transcriptome and contrast resistant and susceptible SCN reactions at 6 and 8 days after inoculation (dai). Our findings reveal likely defense-response gene candidates and a potential regulatory "signature" that captures TFBS over-representation throughout both resistant and susceptible reactions.

\section{Results and discussion}

\section{Illumina sequencing and read alignment}

cDNA libraries from soybean roots were generated after independently inoculating roots for both 6 and 8 dai in two SCN populations, NH1-RHg (confers resistant reaction in Peking; Race 3) and TN8 (confers susceptible reaction in Peking; Race 14). A baseline control cDNA library was also created from roots uninoculated with SCN. RNA was prepared using the Illumina TruSeq sample preparation kit. Single-end RNA-sequencing (RNA-Seq) was performed on the Illumina GAIIx, producing a total of 30 million reads $80 \mathrm{bp}$ in length. Across all sequenced libraries, quality assessment subtracted between 10\%$19 \%$ of reads for being either a contaminent sequence or of low quality (Table 1). Using the BWA aligner [9], quality reads were mapped against the soybean transcriptome build version 1.1 [10]. Reads aligning to multiple transcripts were identified and assigned to the transcript with the highest quality score. In total, 59\% to $69 \%$ of quality-assessed reads mapped to the soybean transcriptome.

\section{Soybean transcript abundance and profiling during SCN pathogenesis}

Differential expression tests were performed using the $\mathrm{R}$ package DESeq [11]. Soybean transcripts were functionally annotated using both Gene Ontology (GO) [12] and PFAM [13]. Both fold change and $\log _{2}$ fold change of expression profiles (as RPKM) were computed between experimental and uninoculated samples. To render a soybean transcript differentially expressed (DE), the transcript had to have a $\log _{2}$ fold change greater than or equal to \pm 1.0 and have atleast 5 mapped reads across all replicates. A total of 12,377 soybean transcripts were identified to be DE in at least one of the samples (Additional file 1). To disseminate the plant-pathogen defenseresponse landscape, a subset of $181 \mathrm{DE}$ transcripts were mined and classified given their GO and PFAM functional annotations (Table 2, Additional file 2). Interestingly, virtually all of these annotation classifications exhibited induced expression profiles exclusive to the resistant reaction. For instance, all 12 transcripts of $\beta-1,4-$ glucanase $(\beta-1,4-\mathrm{G})$ were generally induced throughout the resistant but suppressed in the susceptible reaction. Numerous studies reveal how a pathogenic nematode can commandeer not only $\beta-1,4-$ glucanase but other cellulases to drive formation of a nematode feeding site [14-16]. Both Tucker et al. [16] and Ibrahim et al. [14] quantified this destructive commandeering capability by quantifying the soybean transcriptome using high-throughout microarrays. This latter study, though examining soybean-root knot nematode interplay, reveals cell-wall modeling, defense response, and metabolism, to be the most impacted host pathways following pathogenic nematode infection. Critical genes encoding isoflavonoid and flavonoid biosynthesis such as chalcone synthase (ChS), chalcone reductase (ChR), and chalcone isomerase (ChI) also exhibited similar induced expression profiles. Glutathione S-transferase (GST) genes were also induced in the resistant reaction. GST is a class of enzymes involved in reactions leading to xenobiotic degradation [17], and has been shown to be induced during an SCN resistant reaction [18-20].

Transcripts of genes encoding two lipoxygenase (LOX) gene family members, arachidonate 8-lipoxygenase (A-8 LOX; EC: 1.13.11.40) and linoleate 13S-lipoxygenase (L-13S LOX (LOX2); EC: 1.13.11.12) were also induced throughout both 6 dai and 8 dai resistant reactions. The role A-8 LOX plays during a nematode reaction has yet

Table 1 Soybean-SCN pathogenesis RNA-Seq summary

\begin{tabular}{llllll}
\hline SCN population & Time point & SRA & Reads & Filtered & Aligns to soybean \\
\hline Uninoculated & Control & SRR849499 & $2,141,303$ & $401,913(19 \%)$ & $1,201,664(69 \%)$ \\
\hline \multirow{2}{*}{ Race3 } & 6 dai & SRR847313 & $8,069,844$ & $1,130,372(14 \%)$ & $4,640,251(67 \%)$ \\
& 8 dai & SRR848922 & $7,319,342$ & $745,019(10 \%)$ & $4,135,793(63 \%)$ \\
\hline \multirow{2}{*}{ Race14 } & 6 dai & SRR848921 & $9,160,690$ & $1,624,774(18 \%)$ & $4,486,182(59 \%)$ \\
& 8 dai & SRR849498 & $4,078,344$ & $637,475(15 \%)$ & $2,193,208(63 \%)$ \\
\hline Total & - & - & $30,769,523$ & $4,539,553(14 \%)$ & $16,657,098(63 \%)$ \\
\hline
\end{tabular}

Summary of reads generated throughout a Race 3 and Race 14 SCN inoculation. Low quality reads were subtracted from the total read-set. Remaining reads were then mapped to the soybean transcriptome. 
Table 2 Various genes perceived during defense response are expressed during $\mathrm{SCN}$ inoculation

\begin{tabular}{|c|c|c|c|c|c|}
\hline \multirow[b]{3}{*}{ Function } & \multirow[b]{3}{*}{$n$} & \multicolumn{4}{|c|}{ Median $\log _{2}$ RPKM } \\
\hline & & \multicolumn{2}{|c|}{ Race 3} & \multicolumn{2}{|c|}{ Race 14} \\
\hline & & 6 dai & 8 dai & 6 dai & $8 \mathrm{dai}$ \\
\hline$\beta-1,4-G$ & 12 & 1.14 & 0.85 & 0.27 & 0 \\
\hline $4 \mathrm{CL}$ & 26 & 0.46 & 0.69 & -1.03 & -0.27 \\
\hline A-8 LOX & 18 & 0 & 1.03 & -1.77 & -0.59 \\
\hline ChR & 5 & 1.07 & 1.01 & 0 & -0.53 \\
\hline Chl & 6 & 1.33 & 0.63 & -0.37 & -0.62 \\
\hline ChS & 15 & 1.18 & 1.39 & -0.76 & -0.73 \\
\hline GST & 21 & 1.15 & 1.14 & -1.02 & 0 \\
\hline GLY। & 5 & 1.41 & 1.11 & -1.49 & -1.39 \\
\hline L-13S LOX & 17 & 0.91 & 1.40 & -1.76 & -0.81 \\
\hline PCS & 4 & 0.74 & 1.49 & -1.29 & -0.41 \\
\hline PR5 & 15 & 1.66 & 0.54 & -1.38 & -0.53 \\
\hline PR10 & 15 & 1.31 & 1.12 & -1.16 & -1.23 \\
\hline PDI & 9 & 1.08 & 1.60 & -0.89 & -1.08 \\
\hline $\mathrm{RnDR}$ & 5 & 1.39 & 0 & 0 & 0 \\
\hline SOD & 8 & 1.12 & 0.60 & -0.58 & 0 \\
\hline
\end{tabular}

Numerous genes are involved in defense-response. DE transcripts were binned based on GO or PFAM annotated function, yielding bins of differing size, $n$.

to be elucidated, however lipoxygenases in-general are consistently induced throughout a resistant $\mathrm{SCN}$ reaction [21-24]. This raises speculation that A-8 LOX may be perceived during $\mathrm{SCN}$ pathogenesis.

Ribonucleoside-diphosphate reductase (RnDR; EC: 1.17.4.1) and protein disulfide-isomerase (PDI; EC: 5.3 .4 .1 ) were induced in the resistant reaction. Both RnDR and PDI are thioredoxins, a family of reductases known to play defense-response roles upon perception of a pathogen [25-27]. Little is known about the role RnDR plays in SCN pathogenesis, however an earlier microarray study examined abaxial and adaxial soybean embryo expression profiles upon exposure to auxin 2,4-dichlorophenoxyacetic acid (2,4-D). Microarray results revealed differentially expressed levels of RnDR 21 days after auxin inoculation [28]. PDI on the other hand, is a well-studied thioreductase expressed during plant defense [29,30], especially in soybean roots undergoing a resistant SCN reaction [31].

Pathogenesis-Related (PR) transcripts, namely PR 5 and PR10, were induced in the resistant reaction. PR genes were expressed not just during SCN nematode pathogenesis [32-38] but also throughout abiotic stress [39], phytohormone signaling [40] and drought [41].

Glyoxalase I (GLY I; lactoylglutathione lyase, EC: 4.4.1.5) was also induced throughout the resistant reaction. GLY I has been shown to exhibit an induced expression profile in pumpkin seeds exposed to numerous abiotic stresses [42]. Lastly, little is known about the role phytochelatin synthetase (PCS) plays throughout SCN pathogenesis, however PCS has been shown in a prior study to be induced during aphid herbivory [43].

Following quantification of the SCN-inoculated soybean root transcriptome, our analyses support earlier works by Klink et al. ([44,45]), Kandoth et al. ([20]), and Li et al. ([33]). We build-on such studies by identifying a small subset of potentially novel defense-response candidate genes as well as a biologically-sound proximal regulatory landscape that captures host-SCN pathogenesis interplay.

\section{Gene Ontology enrichment in resistant and susceptible reactions}

To identify statistically significant Gene Ontology (GO) annotations, the top 750 induced and 750 suppressed genes across for all SCN samples each independently underwent GO Process enrichment using the AgriGO server [46]. Numerous GO Processes were statistically significant across resistant and susceptible reactions (Table 3). GO Process $p$-values were adjusted using Bonferroni False Discovery Rate (FDR) and all GO Processes with adjusted $p$-values less than 0.05 were selected.

The top 30 most statistically significant GO Processes within induced genes were identified (Table 4). Processes such as "defense response", "syncytium formation", "response to other organism", "response to oxidative stress", and "response to stress", were revealed to be statistically significant mainly in the resistant reaction when compared to the susceptible. Processes associated with organelle modification and intracellular organization also exhibited similar reaction-specific significance. This race-exclusivity exposes the crucial role basal operations play during pathogen perception.

Similarly, the top 30 most statistically significant GO Processes within suppressed genes were also identified (Table 5). Contrasting GO Processes in suppressed genes to that of induced genes reveals an entirely different catalog of annotations. For instance, 20 of the

Table 3 Abundance of enriched Gene Ontology annotations

\begin{tabular}{|c|c|c|c|c|c|}
\hline & & \multicolumn{2}{|c|}{ Race 3} & \multicolumn{2}{|c|}{ Race 14} \\
\hline & & 6 dai & 8 dai & 6 dai & 8 dai \\
\hline \multirow{2}{*}{ Count } & Induced & 53 & 48 & 25 & 19 \\
\hline & Suppressed & 73 & 104 & 113 & 86 \\
\hline
\end{tabular}

Enriched GO annotations throughout each inoculation. Per inoculation, the top-750 induced and top-750 suppressed DE transcripts were identified and enriched GO annotations were identified. Only enrichments with a Bonferroni-corrected $p$-value less than 0.05 were selected. Counts represent both GO Process and GO Function. 
Table 4 GO Process enrichment of induced soybean genes

\begin{tabular}{|c|c|c|c|}
\hline \multirow[b]{2}{*}{ Term } & \multirow[b]{2}{*}{ Description } & \multicolumn{2}{|c|}{$-\log _{10} F D R$} \\
\hline & & Race 3 & Race 14 \\
\hline GO:0042545 & Cell wall modification & 10.49 & 0 \\
\hline GO:0042547 & $\begin{array}{l}\text { Cell wall modification during } \\
\text { multidimensional cell growth }\end{array}$ & 10.52 & 4.25 \\
\hline GO:0044085 & Cellular component biogenesis & 3.20 & 0 \\
\hline GO:0034622 & $\begin{array}{l}\text { Cellular macromolecular complex } \\
\text { assembly }\end{array}$ & 4.25 & 0 \\
\hline GO:0046916 & $\begin{array}{l}\text { Cellular transition metal ion } \\
\text { homeostasis }\end{array}$ & 0 & 4.52 \\
\hline GO:0031497 & Chromatin assembly & 11.74 & 0 \\
\hline GO:0006333 & $\begin{array}{l}\text { Chromatin assembly or } \\
\text { disassembly }\end{array}$ & 10.18 & 0 \\
\hline GO:0006325 & Chromatin organization & 7.18 & 0 \\
\hline GO:0051276 & Chromosome organization & 6.11 & 0 \\
\hline GO:0006952 & Defense response & 6.69 & 1.45 \\
\hline GO:0006323 & DNA packaging & 11.55 & 0 \\
\hline GO:0065003 & $\begin{array}{l}\text { Macromolecular complex } \\
\text { assembly }\end{array}$ & 3.92 & 0 \\
\hline GO:0051704 & Multi-organism process & 3.69 & 0 \\
\hline GO:0009825 & Multidimensional cell growth & 6.08 & 1.79 \\
\hline GO:0006334 & Nucleosome assembly & 12.92 & 0 \\
\hline GO:0034728 & Nucleosome organization & 11.85 & 0 \\
\hline GO:0006996 & Organelle organization & 3.48 & 0 \\
\hline GO:0010117 & Photoprotection & 4.56 & 0 \\
\hline GO:0009828 & Plant-type cell wall loosening & 8.40 & 2.56 \\
\hline GO:0009827 & Plant-type cell wall modification & 8.56 & 0 \\
\hline GO:0009831 & $\begin{array}{l}\text { Plant-type cell wall modification } \\
\text { during multidimensional cell } \\
\text { growth }\end{array}$ & 6.38 & 2.02 \\
\hline GO:0009664 & Plant-type cell wall organization & 6.25 & 0 \\
\hline GO:0065004 & Protein-DNA complex assembly & 12.40 & 0 \\
\hline GO:0009725 & Response to hormone stimulus & 2.50 & 5.95 \\
\hline GO:0051707 & Response to other organism & 5.21 & 1.88 \\
\hline GO:0006979 & Response to oxidative stress & 10.66 & 0 \\
\hline GO:0006950 & Response to stress & 5.35 & 0 \\
\hline GO:0006949 & Syncytium formation & 7.45 & 2.43 \\
\hline GO:0055076 & Transition metal ion homeostasis & 0 & 4.52 \\
\hline GO:0006414 & Translational elongation & 5.16 & 0 \\
\hline
\end{tabular}

GO Process enrichment from the top 750 induced transcripts. Numerous GO Processes associated with cell-wall modification, intracellular organization and defense response exhibit increased enrichment during the resistant reaction.

30 GO Processes in suppressed genes are statistically significant across both resistant and susceptible reactions. This indicates that nematode effectors are generally operable in a race-independent manner and capable of effortlessly suppressing a majority of crucial basal processes.
Table 5 GO Process enrichment of suppressed soybean genes

\begin{tabular}{|c|c|c|c|}
\hline \multirow[b]{2}{*}{ Term } & \multirow[b]{2}{*}{ Description } & \multicolumn{2}{|c|}{$-\log _{10} F D R$} \\
\hline & & Race 3 & Race 14 \\
\hline GO:0006066 & Alcohol metabolic process & 0 & 2.52 \\
\hline GO:0016051 & $\begin{array}{l}\text { Carbohydrate biosynthetic } \\
\text { process }\end{array}$ & 4.56 & 7.92 \\
\hline GO:0044262 & $\begin{array}{l}\text { Cellular carbohydrate metabolic } \\
\text { process }\end{array}$ & 0 & 2.17 \\
\hline GO:0043094 & $\begin{array}{l}\text { Cellular metabolic compound } \\
\text { salvage }\end{array}$ & 2.88 & 5.53 \\
\hline GO:0006091 & $\begin{array}{l}\text { Generation of precursor } \\
\text { metabolites and energy }\end{array}$ & 83.18 & 87.31 \\
\hline GO:0006544 & Glycine metabolic process & 2.20 & 0 \\
\hline GO:0006096 & Glycolysis & 1.48 & 3.95 \\
\hline GO:0018130 & Heterocycle biosynthetic process & 6.42 & 4.20 \\
\hline GO:0019318 & Hexose metabolic process & 1.79 & 5.33 \\
\hline GO:0042743 & $\begin{array}{l}\text { Hydrogen peroxide metabolic } \\
\text { process }\end{array}$ & 2.66 & 0 \\
\hline GO:0006555 & Methionine metabolic process & 2.12 & 0 \\
\hline GO:0006740 & $\mathrm{NADPH}$ regeneration & 0 & 2.37 \\
\hline GO:0006733 & $\begin{array}{l}\text { Oxidoreduction coenzyme } \\
\text { metabolic process }\end{array}$ & 0 & 2.92 \\
\hline GO:0009853 & Photorespiration & 6.48 & 9.04 \\
\hline GO:0015979 & Photosynthesis & 215.70 & 211.61 \\
\hline GO:0009765 & Photosynthesis, light harvesting & 81.37 & 68.25 \\
\hline GO:0009768 & $\begin{array}{l}\text { Photosynthesis, light harvesting } \\
\text { in photosystem I }\end{array}$ & 52.95 & 39.57 \\
\hline GO:0019684 & Photosynthesis, light reaction & 132.78 & 130.48 \\
\hline GO:0009767 & $\begin{array}{l}\text { Photosynthetic electron transport } \\
\text { chain }\end{array}$ & 43.33 & 47.11 \\
\hline GO:0009773 & $\begin{array}{l}\text { Photosynthetic electron transport } \\
\text { in photosystem I }\end{array}$ & 23.73 & 28.55 \\
\hline GO:0042549 & Photosystem II stabilization & 4.76 & 9.29 \\
\hline GO:0046148 & Pigment biosynthetic process & 8.81 & 11.14 \\
\hline GO:0042440 & Pigment metabolic process & 14.26 & 17.96 \\
\hline GO:0018298 & Protein-chromophore linkage & 51.69 & 42.96 \\
\hline GO:0043467 & $\begin{array}{l}\text { Regulation of generation of } \\
\text { precursor metabolites and energy }\end{array}$ & 1.88 & 4.38 \\
\hline GO:0042542 & Response to hydrogen peroxide & 0 & 5.20 \\
\hline GO:0010035 & Response to inorganic substance & 0 & 6.25 \\
\hline GO:0009416 & Response to light stimulus & 11.30 & 13.85 \\
\hline GO:0009314 & Response to radiation & 10.71 & 13.19 \\
\hline GO:0000302 & $\begin{array}{l}\text { Response to reactive oxygen } \\
\text { species }\end{array}$ & 0 & 3.73 \\
\hline
\end{tabular}

GO Process enrichment from the top 750 suppressed transcripts. Almost all GO Processes were suppressed in a race-independent manner. The suppressive cocktail of SCN effectors are revealed in the down-regulation of processes associated with photosynthesis, metabolism and biosynthesis. 
The most suppressed GO Processes were "photosynthesis", "photosynthesis, light harvesting", "photosynthesis, light reaction", and "generation of precursor metabolites and energy". Interestingly, it has been shown in prior studies that PPNs can suppress photosynthesis in tomato plants by disrupting cytokinin and gibberellin signaling $[47,48]$. Aside from photosynthetic processes, those associated with metabolism and biosynthesis were highly suppressed across both reactions. This suggests that both resistant and susceptible $\mathrm{SCN}$ populations share a common goal of crippling basal metabolic machinery and suppressing the host machinery responsible for photosynthesis.

\section{Derivation of over-represented TFBSs}

The 1,000 most induced and 1,000 most suppressed genes were identified for each sample and the promoter sequence $2 \mathrm{~kb}$ upstream from each genes transcription start site was retrieved and appended to a FASTA file (Additional file 3). To quantify abundance of cisregulatory TFBSs within promoter sequences, we used a collection of 68 plant Position Weight Matrices (PWMs) from AthaMap [49] and JASPAR [50]. PWMs are multidimensional matrices frequently used to model regulatory elements, namely TFBSs. Each cell in a PWM represents a weight as to the likelihood a particular base at a specific index is a regulatory element. Thus, mapping PWMs onto promoter sequences and statistically quantifying its abundance reveals insight into the magnitude of TFBS overrepresentation. To efficiently execute such mapping, we had developed a multivariate statistical software named
Marina [51]. Marina maps TFBS models such as PWMs onto promoter sequences and infers magnitude of TFBS over-representation using 7 knowledge-discovery metrics. The Iterative Proportional Fitting (IPF) algorithm [52] normalizes output produced from each of the 7 metrics, enabling unanimous agreement across the metrics as to the magnitude of TFBS over-representation. IPF scores range from 1 to $N$ whereby $N$ is the total number of overrepresented TFBSs. Scores in the range of 1 represent over-represented TFBSs while scores in the range of $N$ represent highly under-represented TFBSs.

For all SCN samples, Marina mapped all 68 plant PWMs onto promoter sequences of both induced and suppressed genes. In total, 46 TFBSs were over-represented in atleast one of the four samples (Figure 1). To reveal which TFBSs exhibited variations in their IPF scores, we computed the percent change of IPF scores across both Race 3 and Race 14 timepoints. The difference in Race 3 and Race 14 percent change was derived and partitioned into 2 bins: TFBSs with a Race 3 and Race 14 IPF score percent difference of at least 50\% (Figure 1a), and TFBSs with a Race 3 and Race 14 IPF score percent difference under 50\% (Figure 1b). Thus, such computation allows for identification of which TFBSs vary greatly not with respect to 6 dai or 8 dai, but with respect to Race 3 and Race 14 inoculations.

There were 29 TFBSs over-represented across all four samples (Additional file 4). If a TFBS was not overrepresented in a specific sample, that TFBS was assigned an score of $N+1$ so as to serve as a proxy for being highly under-represented.

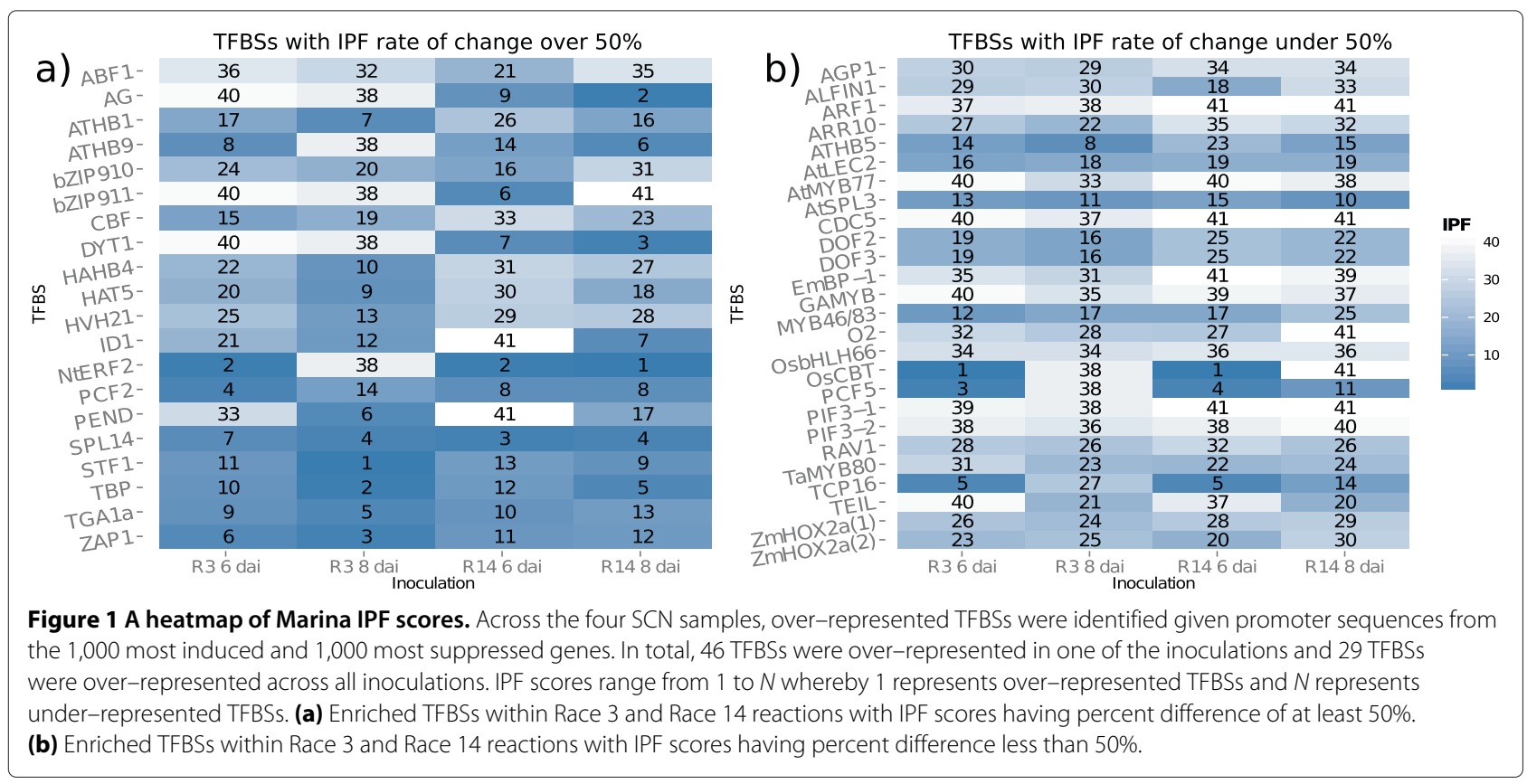




\section{Many TFBSs are over/under-represented in both resistant and susceptible reactions}

Contrasting TFBS IPF scores across samples reveals that 30 of the 46 TFBSs either increase or decrease in IPF score regardless of the reaction (Figure 1). For instance, the TFBS for STF1 exhibits a relatively modest increase in its IPF score across both reactions. Interestingly, STF1 IPF score increases from 11th to 1st from 6 dai to 8 dai respectively in the resistant reaction. Besides the role STF1 plays in plant development [53], little is known of the role this transcription factor plays in plant defense.

IPF score for the HAHB4 TFBS greatly increased in the resistant reaction and susceptible reaction. A prior study found HAHB4 to contribute to jasmonic acid and ethylene signaling crosstalk [54]. Similarly, TFBSs for DOF2 and DOF3 exhibited relatively weak increases in IPF scores across resistant and susceptible samples. DOF transcripts have not been explicitly quantified as-far as their gene expression during SCN pathogenesis, however such proteins have been detected during auxin signaling [55]. In contrast to DOF2 and DOF3, the TFBS for TEIL had a near-50\% jump in IPF scores across both reactions. Being the tobacco homolog of ethylene insensitive (EIN3), TEIL gene products have been shown to bind directly to the promoter sequence of PR1a, a central contributor in plant defense dynamics [56]. Interestingly, across both resistant and susceptible reactions, TEIL scores appear to be relatively equal to one another.

The A. thaliana MYB77 homolog, AtMYB77, exhibits a mild change in IPF score across both resistant and susceptible reactions. Across both reactions, AtMYB77 IPF scores were generally under-represented at 6 dai but become slightly over-represented at 8 dai. An earlier study revealed interaction between MYB77 and auxin response factor 7 (ARF7) [57], further accentuating the role AtMYB77 could play in host-pathogen interplay [58]. The OsCBT TFBS exhibited pronounced IPF scores across all four treatments. In both the resistant and susceptible reaction, OsCBT was highly over-represented only at 6 dai. It was shown that OsCBT mutants conferred increased pathogen resistance upon inoculation with Magnaporthe grisea, revealing that OsCBT suppresses defense response [59].

\section{Several TFBSs are over-represented in a race-dependent manner}

The remaining 16 TFBSs were over-represented in one reaction compared to the other. Such TFBSs can expose novel insight into TFBSs over-representation patterns respective to a specific reaction.

ZAP1, a WRKY1 TFBS [60], appears to be highly over-represented during the resistant reaction but slightly under-represented in the susceptible reaction. Being a WRKY TFBS, it comes as no surprise that enrichment of this TFBS in the resistant reaction captures the need to host a significant, systematic plant defense response. Similarly, PIF3-1 and PIF3-2 were both under-represented during the susceptible reaction however slightly overrepresented in the resistant reaction. It has been shown that PIF plays roles in phytochrome signaling [61]. Due to its photomorphogenic regulatory capabilities, Since photosynthetic processes are heavily suppressed within resistant and susceptible reactions (Table 5), such suppression explains why PIF3-1 and PIF3-2 have such severely under-represented IPF scores. Indeed SCN pathogenesis does not only disrupt the photosynthetic machinery but also the plants ability to execute sound phytochrome signaling.

\section{Conclusions}

We used RNA-Seq to sequence soybean whole-root (Peking cv.) at both 6 and 8 dai upon inoculation with a resistant (NH1-RHg; Race 3) and susceptible (TN8; Race 14) population. Contrasting TFBSs over-represented in promoter sequences of DE soybean genes across 6 and 8 dai time points exposed underlying transcriptomic and cis-regulatory dynamics within the soybean root during pathogenesis. In-total, over 30 million reads from soybean whole-root was sequenced and differential expression analysis revealed 181 transcripts to be statistically and biologically significant during defense-response. Several viable defense-response gene candidates joined these ranks, including glyoxalase I, arachidonate-8 lipoxygenase, phytochelatin synthetase, and ribonucleosidediphosphate reductase.

46 TFBSs were rendered over/under-represented across all resistant and susceptible samples. Interestingly, 30 of these TFBSs were either over or under-represented across both reactions. Thus, our results reveal presence of a biologically-sound regulatory "signature" that identifies reaction-specific soybean regulatory patterns during both resistant and susceptible SCN reactions.

\section{Methods}

\section{Plant procurement and SCN inoculation}

Glycine max cv. Peking seeds were surface-sterilized by treating the seeds with $10 \%$ bleach $(0.6 \%$ sodium hypochlorite) for ten minutes, followed by several washes with distilled water. Seeds were planted in sterile sand in $20 \times 20 \mathrm{~cm}$ flats. Eight days later, seedlings were gently lifted out of the sand and rinsed clean. Five seedlings for each time point were placed on moistened germination paper in $8 \times 12 \times 3.5 \mathrm{~cm}$ plastic trays. The $\mathrm{SCN}$ populations NH1-RHg and TN8, were independently harvested from stock plants [62]. Females were crushed with a rubber stopper and eggs were washed through a 
250 micron screen and collected on a 25 micron screen. Eggs were rinsed into a small covered tray and left to hatch for three days. J2 stage nematodes were further purified by passing them through a 30 micron cloth into deionized, distilled water and gently centrifuged at 250 relative centrifugal force (RCF) for one minute to concentrate to 2,000 $\mathrm{J} 2 / \mathrm{ml}$. Roots from four plants were inoculated with one $\mathrm{ml}$ of inoculum. Roots were covered with a second piece of moistened germination paper and the trays were placed in a larger tray with $0.5 \mathrm{~cm}$ water below to add humidity and wrapped in a semiclear plastic bag for the duration of the time points. Three uninoculated control plants were also placed trays and collected separately. Per plant, four plant roots, following 6 and 8 days after inoculation (dai), were harvested and immediately frozen in liquid nitrogen and ground to a fine powder in a mortar and pestle and stored in microfuge tubes at $-80^{\circ} \mathrm{C}$ until RNA extraction. The fifth root was stained for visualization of nematode infection with acid fuchsin [63]. RNA was extracted at 6 dai and 8 dai by phenol/chloroform and lithium chloride precipitation [64]. RNA was treated with DNase to remove any genomic DNA remaining in the samples. RNA integrity was checked by visualizing the intact $18 \mathrm{~S}$ and $28 \mathrm{~S}$ ribosomal bands on an agarose gel and concentrations were measured on a Nanodrop spectrophotometer (Thermo Scientific; Waltham, MA).

\section{RNA extraction and CDNA isolation}

cDNA libraries were prepared using the TruSeq RNA Prep Kit according to the manufacturer instruction (Illumina). Briefly, mRNA was purified from four micrograms of total RNA diluted in fifty microliters of nuclease-free ultra pure water using magnetic beads. Resulting mRNA was fragmented at $94^{\circ} \mathrm{C}$ for eight minutes. Seventeen microliters of fragmented mRNA was used as template for cDNA synthesis performed by a Superscript II Reverse Transcriptase. Second-strand synthesis was immediately performed and fifty microliters of double stranded DNA was transferred to a new tube and submitted to end repair followed by adenylation of 3 ' ends. Once adenylation of 3' reached completion, adapters containing different indexes were ligated to each library. DNA fragments having adapter molecules on both ends were amplified and enriched. Quantification and quality control were performed by loading one microliter of cDNA libraries on an Agilent DNA-1000 chip and running it on an Agilent Technologies 2100 Bioanalyzer.

\section{Deep-sequencing and transcriptome quantification}

For both NH1-RHg (Race 3) and TN8 (Race 14) reactions, cDNA libraries were sequenced from 8 day old soybean whole-root independently inoculated with SCN at 6 dai and 8 dai. Two biological replicates were sequenced for each inoculation and timepoint. Singleend RNA-sequencing was performed on the Illumina GAIIx at the United States Department of Agriculture (USDA), Beltsville, MD. An uninoculated whole-root single-replicate control was also sequenced using the same sequencing protocol. To remove low quality reads across all sequencing runs, custom bash scripts filtered all reads should its 3' tail have a quality score of less than 22 . To remove contaminent reads, sequences were subtracted if they mapped atleast once to both the Ensembl human genome (Hg19) or the JCVI Microbial Resource [65]. Remaining sequences were mapped to the soybean transcriptome (build 1.1) using BWA [9]. Across all SCN inoculated samples, transcript counts underwent normalization and variance estimation using the DESeq R package. To infer magnitude of differential expression, RPKM was computed for all inoculated and uninoculated samples and $\log _{2}\left(\frac{R P K M_{\text {inoculated }}}{R P K M_{\text {uninoculated }}}\right)$ was subsequently derived. All transcripts with a $\log _{2}$ RPKM less than 1 and fewer than 5 mapped reads were rendered not differentially expressed.

\section{Functional annotation \& Gene Ontology (GO) enrichment}

Functional annotation comprised of homology-based analysis of all sequences in the Phytozome soybean transcriptome. Of these 73,320 soybean transcriptome sequences, 7,810 sequences were subtracted for being either a scaffold or duplicate sequence. BLASTX [66] aligned the remaining 65,510 query sequences onto all UniProt plant proteins [67]. The top-scoring UniProt function annotation was assigned to the query if it did not contain ambiguous keywords, namely "Hypothetical", "Uncharacterized" or "Unknown".

For all samples, soybean Phytozome accessions for the top 750 induced and top 750 suppressed transcripts were identified. Gene Ontology (GO) enrichment on each accession-set was performed using the AgriGO web-server [46]. AgriGO settings were modified to quantify $\mathrm{GO}$ annotations using the hypergeometric distribution and Bonferroni $p$-value false-discovery rate (FDR) correction. To measure GO Process statistical significance in both resistant and susceptible reactions, the $-\log _{10} F D R$ per GO Process was summed across both 6 and 8 dai time points. Subsequently, the top 30 most statistically significant GO Processes from the top 750 induced and suppressed transcript sets were identified.

\section{Availability of supporting data}

All RNA-Seq FASTQ raw data is available from NCBI SRA. Please refer to Table 1 for such accessions. 


\section{Additional files}

Additional file 1: Differentially expressed transcripts across al inoculations. A table of 12,377 transcripts that are DE across all four SCN inoculations.

Additional file 2: Differentially expressed transcripts annotated to be involved in plant defense. A set of 181 transcripts collectively annotated by $G O$ and PFAM annotations to contribute to plant defense.

Additional file 3: TFBSs over-represented across all inoculations. A collection of 46 TFBSs over-represented in atleast one inoculation.

Additional file 4: Promoter sequences of induced and suppressed transcripts. FASTA sequences representing promoter sequences of induced and suppressed transcripts following 6 dai and 8 dai with SCN virulent and avirulent populations.

\section{Abbreviations}

4CL: 4-Coumarate-CoA ligase; A-8 LOX: Arachidonate 8-lipoxygenase; L-13S LOX: Linoleate 13S-lipoxygenase; Chl: Chalcone isomerase; ChR: Chalcone reductase; GST: Glutathione S-transferase; GLY I: Glyoxalase I; GO: Gene Ontology; PCS: Phytochelatin synthetase; PDI: Protein disulfide-isomerase; PPN: Plant Parasitic Nematode; PR: Pathogenesis-related; PWM: Position weight matrix; RnDR: Ribonucleoside-diphosphate reductase; SOD: Super-oxide dismutase; SCN: Soybean cyst nematode; STF1: Starch-Free 1; TF: Transcription factor; TFBS: Transaction factor binding site.

Competing interests

The authors declare that they have no competing interests.

\section{Authors' contributions}

$\mathrm{PH}$ wrote the manuscript, performed RNA-Seq analysis and quantification of over-represented TFBSS. BFM conceived of the study and oversaw TFBS quantification. Both authors read, critiqued and approved the final manuscript.

\section{Acknowledgements}

We wish to thank the United States Department of Agriculture - Soybean Genomics and Improvement Laboratory (USDA - SGIL) for research funding and support. Our appreciations go out to Ivan Ovcharenko for advice on GO enrichment analysis and TFBS over-representation derivation. We wish to thank Arianne Tremblay for overseeing cDNA derivation and RNA extraction. We wish to thank Margaret H. MacDonald for inoculation of soybean roots with SCN. We also wish to thank Patrick Gillevet and James Willett for numerous thought-provoking discussions. This research was supported in part by the Intramural Research Program of the National Institutes of Health, National Library of Medicine.

\section{Author details \\ ${ }^{1}$ School of Systems Biology, George Mason University, Manassas, VA, USA. ${ }^{2}$ Computational Biology Branch, National Center for Biotechnology Information, National Institutes of Health, Bethesda, MD, USA. ${ }^{3}$ Soybean Genomics and Improvement Laboratory, United States Department of Agriculture, Beltsville, MD, USA.}

Received: 5 June 2013 Accepted: 22 October 2014

Published online: 25 November 2014

\section{References}

1. Wrather J, Anderson T, Arsyad D, Tan Y, Ploper L, Porta-Puglia A, HH R, Yorinori J: Soybean disease loss estimates for the top ten soybean-producing counries in 1998. Can J Plant Pathol 2001 , 23(2):115-121.

2. Matsye P, Lawrence G, Youssef R, Kim K, Lawrence K, Matthews B, Klink V: The expression of a naturally occurring, truncated allele of an $\alpha$-SNAP gene suppresses plant parasitic nematode infection. Plant Mol Biol 2012, 80(2):131-155.

3. Endo B: Penetration and development of Heterodera glycines in soybean roots and related anatomical changes. Phytopath 1964 , 54:79-88
4. Klink V, Hosseini $P$, MacDonald M, Alkharouf N, Matthews B: Population-specific gene expression in the plant pathogenic nematode Heterodera glycines exists prior to infection and during the onset of a resistant or susceptible reaction in the roots of the Glycine max genotype Peking. BMC Genomics 2009, 10:111.

5. Li X, Wang X, Zhang S, Liu D, Duan Y, Dong W: Comparative profiling of the transcriptional response to soybean cyst nematode infection of soybean roots by deep sequencing. Chin SciBull 2011 , 56(18):1904-1911.

6. Li X, Wang X, Zhang S, Liu D, Duan Y, Dong W: Identification of soybean micrornas involved in soybean cyst nematode infection by deep sequencing. PLOS ONE 2012, 7(6):e39650.

7. Hamamouch N, Li C, Hewezi T, Baum T, Mitchum M, Hussey R, Vodkin L, Davis $E$ : The interaction of the novel $30 \mathrm{CO} 2$ cyst nematode effector protein with a plant $\boldsymbol{\beta}$-1,3-endoglucanase may suppress host defence to promote parasitism. J Exp Bot 2012, 63(10):3683-3695.

8. Guttikonda S, Trupti N, Bisht J, Chen H, An Y, Pandey D, Xu S, Yu O: Whole genome co-expression analysis of soybean cytochrome $P 450$ genes identifies nodulation-specific P450 monooxygenases. BMC Plant Biol 2010, 10:243.

9. Li H, Durbin R: Fast and accurate short read alignment with Burrows-Wheeler transform. Bioinformatics 2009, 25(14):1754-1760.

10. Goodstein DM, Shu S, Howson R, Neupane R, Hayes RD, Fazo J, Mitros T, Dirks W, Hellsten U, Putnam N, Rokhsar DS: Phytozome: a comparative platform for green plant genomics. Nucleic Acids Res 2012, 40(Database issue):D1178—D1186.

11. Anders $S$, Huber W: Differential expression analysis for sequence count data. Genome Biol 2010, 11(10):R106.

12. Ashburner M, Ball CA, Blake JA, Botstein D, Butler H, Cherry JM, Davis AP, Dolinski K, Dwight SS, Eppig JT, Harris MA, Hill DP, Issel-Tarver L, Kasarskis A, Lewis S, Matese JC, Richardson JE, Ringwald M, Rubin GM, Sherlock G: Gene Ontology: tool for the unification of biology. Nat Genet 2000, 25:25-29.

13. Bateman A, Birney E, Cerruti L, Durbin R, Etwiller L, Eddy SR, Griffiths-Jones S, Howe KL, Marshall M, Sonnhammer ELL: The Pfam Protein Families Database. Nucleic Acids Res 2002, 30:276-280.

14. Ibrahim HM, Hosseini P, Alkharouf NW, Hussein EH, Gamal ED AEKE, Aly MA, Matthews BF: Analysis of gene expression in soybean (Glycine max) roots in response to the root-knot nematode Meloidogyne incognita using microarrays and KEGG pathways. BMC Genomics 2011, 12:220.

15. Goellner $M$, Wang $X$, Davis E: Endo- $\boldsymbol{\beta}-\mathbf{1}, \mathbf{4}$-glucanase expression in compatible plant-nematode interactions. Plant Cell 2001, 13(10):2241-2255

16. Tucker M, Burke A, Murphy C, Thai V, Ehrenfried M: Gene expression profiles for cell wall-modifying proteins associated with soybean cyst nematode infection, petiole abscission, root tips, flowers, apical buds, and leaves. J Exp Bot 2007, 58(12):3395-3406.

17. Dalton D, Boniface C, Turner Z, Lindahl A, Kim H, Jelinek L, Govindarajulu M, Finger R, Taylor C: Physiological roles of glutathione s-transferases in soybean root nodules. Plant Physio/ 2009, 150:521-530.

18. Mazarei M, Liu W, Al-Ahmad H, Arelli P, Pantalone V, Stewart CJ: Gene expression profiling of resistant and susceptible soybean lines infected with soybean cyst nematode. Theor App/ Genet 2011, 123(7):1193-1206

19. Alkharouf N, Khan R, Matthews B: Analysis of expressed sequence tags from roots of resistant soybean infected by the soybean cyst nematode. Genome 2004, 47(2):380-388.

20. Kandoth P, Ithal N, Recknor J, Maier T, Nettleton D, Baum T, Mitchum M: The Soybean Rhg1 locus for resistance to the soybean cyst nematode Heterodera glycines regulates the expression of a large number of stress- and defense-related genes in degenerating feeding cells. Plant Physiol 2011, 155(4):1960-1975.

21. Klink VP, Matthews BF: Emerging approaches to broaden resistance of soybean to soybean cyst nematode as supported by gene expression studies. Plant Physiol 2009, 151(3):1017-1022.

22. Klink VP, Matsye PD, Lawrence KS, Lawrence GW: Engineered soybean cyst nematode resistance. In Soybean - Pest Resistance. Edited by El-Shemy H: InTech; 2013. doi:10.5772/54514. ISBN: 978-953-51-0978-5, Available from: [http://www.intechopen.com/books/soybean-pestresistance/engineered-soybean-cyst-nematode-resistance] 
23. Veronico P, Giannino D, Melillo M, Leone A, Reyes A, Kennedy M, Bleve-Zacheo T: A novel lipoxygenase in pea roots: its function in wounding and biotic stress. Plant Physiol 2006, 141(3):1045-1055.

24. Ithal N, Recknor J, Nettleton D, Maier T, Baum TJ, Mitchum MG: Developmental transcript profiling of cyst nematode feeding cells in soybean roots. Mol Plant Microbe Interact 2007, 20(5):510-525.

25. Vieira Dos Santos C, Rey P: Plant thioredoxins are key actors in the oxidative stress response. Trends Plant Sci 2006, 11(7):329-334.

26. Laloi C, Mestres-Ortega D, Marco Y, Meyer Y, Reichheld J: The Arabidopsis cytosolic thioredoxin $\mathrm{h} 5$ gene induction by oxidative stress and its W-box-mediated response to pathogen elicitor. Plant Physiol 2004, 134(3):1006-1016.

27. Wang D, Weaver ND, Kesarwani M, Dong X: Induction of protein secretory pathway is required for systemic acquired resistance. Science 2005, 308(5724):1036-1040.

28. Thibaud-Nissen F, Shealy R, Khanna A, Vodkin L: Clustering of microarray data reveals transcript patterns associated with somatic embryogenesis in soybean. Plant Physio/ 2003, 132:118-136.

29. Ray S, Anderson J, Urmeev F, Goodwin S: Rapid induction of a protein disulfide isomerase and defense-related genes in wheat in response to the hemibiotrophic fungal pathogen Mycosphaerella graminicola. Plant Mol Biol 2003, 53(5):701-714.

30. Gruber C, Cemazar M, Clark R, Horibe T, Renda R, Anderson M, Craik D: A novel plant protein-disulfide isomerase involved in the oxidative folding of cystine knot defense proteins. J Biol Chem 2007, 282(28):20435-20446.

31. Klink V, Hosseini $P$, Matsye $P$, Alkharouf N, Matthews B: Syncytium gene expression in Glycine max([PI 88788]) roots undergoing a resistant reaction to the parasitic nematode Heterodera glycines. Plant Physiol Biochem 2010, 48(2-3):176-193

32. Afzal A, Natarajan A, Saini N, labal M, Geisler M, El Shemy H, Mungur R, Willmitzer $L$, Lightfoot $D$ : The nematode resistance allele at the rhg1 locus alters the proteome and primary metabolism of soybean roots. Plant Physiol 2009, 151(3):1264-1280.

33. Li X, Wang X, Zhang S, Liu D, Duan Y, Dong W: Comparative profiling of the transcriptional response to soybean cyst nematode infection of soybean roots by deep sequencing. Chin Sci Bull 2011, 56(18):1904-1911.

34. Matthews BF, Ibrahim HMM, Klink VP: Changes in the expression of genes in soybean roots infected by nematodes. In Soybean - Genetics and Novel Techniques for Yield Enhancement: InTech; 2011 doi:10.5772/20883, ISBN: 978-953-307-721-5. Available from: [http://www. intechopen.com/books/soybean-genetics-and-novel-techniques-foryield-enhancement/changes-in-the-expression-of-genes-in-soybeanroots-infected-by-nematodes]

35. Alkharouf N, Klink V, Chouikha I, Beard H, MacDonald M, Meyer S, Knap H, R K, Matthews B: Timecourse microarray analyses reveal global changes in gene expression of susceptible Glycine max (soybean) roots during infection by Heterodera glycines (soybean cyst nematode). Planta 2006, 224(4):838-852.

36. Ithal N, Recknor J, Nettleton D, Hearne L, Maier T, Baum T, Mitchum M: Parallel genome-wide expression profiling of host and pathogen during soybean cyst nematode infection of soybean. Mol Plant Microbe Interact 2007, 20(3):293-305.

37. Mitchum MG, Baum TJ: Genomics of the soybean cyst nematode-soybean interaction. In Genetics and Genomics of Soybean, Volume 2 of Plant Genetics and Genomics: Crops and Models. Edited by Stacey G. New York: Springer; 2008:321-341.

38. Matthews $B$, Beard $H$, MacDonald MH, Kabir S, Youssef RH, Hosseini $P$, Brewer E: Engineered resistance and hypersusceptibility through functional metabolic studies of 100 genes in soybean to its major pathogen, the soybean cyst nematode. Planta 2013 237(5):1337-1357.

39. Hashimoto M, Kisseleva L, Sawa S, Furukawa T, Komatsu S, Koshiba T: A novel rice PR10 protein, RSOsPR10, specifically induced in roots by biotic and abiotic stresses, possibly via the jasmonic acid signaling pathway. Plant Cell Physiol 2004, 45(5):550-559.

40. Kitajima S, Sato F: Plant pathogenesis-related proteins: molecular mechanisms of gene expression and protein function. J Biochem 1999, 125:1-8.
41. Dubos C, Plomion C: Drought differentially affects expression of a PR-10 protein, in needles of maritime pine (Pinus pinaster Ait.) seedlings. J Exp Bot 2001, 52(358):1143-1144.

42. Hossain MA, Hossain MZ, Fujita M: Stress-induced changes of methylglyoxal level and glyoxalase I activity in pumpkin seedlings and cDNA cloning of glyoxalase I gene. Austr JCrop Sci 2009, 3(2):53-64.

43. Michael Smith C, Boyko EV: The molecular bases of plant resistance and defense responses to aphid feeding: current status. Entomologia Experimentalis et Applicata 2007, 122:1-16.

44. Klink VP, Hosseini P, Matsye P, Alkharouf NW, Matthews BF: A gene expression analysis of syncytia laser microdissected from the roots of the Glycine max (soybean) genotype PI 548402 (Peking) undergoing a resistant reaction after infection by Heterodera glycines (soybean cyst nematode). Plant Mol Biol 2009, 71(6):525-567.

45. Klink VP, Overall CC, Alkharouf NW, MacDonald MH, Matthews BF: A time-course comparative microarray analysis of an incompatible and compatible response by Glycine max (soybean) to Heterodera glycines (soybean cyst nematode) infection. Planta 2007, 226(6):1423-1447.

46. Du Z, Zhou X, Ling Y, Zhang Z: Su Z: agriGO: a GO analysis toolkit for the agricultural community. Nucleic Acids Res 2010, 38(Web-Server-Issue):64-70.

47. Brueske C, Bergeson G: Investigation of growth hormones in xylem exudate and root tissue of tomato infected with root-knot nematode. J Exp Bot 1972, 23(74):14-22

48. Loveys BR, Bird AF: The influence of nematodes on photosynthesis in tomato plants. Physiol Plant Pathol 1973, 3(4):525-529.

49. Bülow L, Engelmann S, Schindler M, Hehl R: AthaMap, integrating transcriptional and post-transcriptional data. Nucleic Acids Res 2009, 37(Database-Issue):D983_D986.

50. Sandelin A, Alkema W, Engstrom P, Wasserman WW, Lenhard B: JASPAR: an open-access database for eukaryotic transcription factor binding profiles. Nucleic Acids Res 2007, 32:D91_D94.

51. Hosseini P, Ovcharenko I, Matthews BF: Using an ensemble of statistical metrics to quantify large sets of plant transcription factor binding sites. Plant Methods 2013, 9:12.

52. Deming WE, Stephan FF: On a least squares adjustment of a sampled frequency table when the expected marginal totals are known. Ann Math Stat 1940, 11(4):427-444.

53. Cheong YH, Yoo CM, Park JM, Ryu GR, Goekjian VH, Nagao RT, Key JL, Cho MJ, Hong JC: STF1 is a novel TGACG-binding factor with a zinc-finger motif and a bZIP domain which heterodimerizes with GBF proteins. Plant J 1998, 15(2):199-209.

54. Manavella PA, Dezar CA, Bonaventure G, Baldwin IT, Chan RL: HAHB4, a sunflower HD-Zip protein, integrates signals from the jasmonic acid and ethylene pathways during wounding and biotic stress responses. Plant J 2008, 56(3):376-388.

55. Yanagisawa S: Dof domain proteins: plant-specific transcription factors associated with diverse phenomena unique to plants. Plant Cell Physiol 2004, 45(4):386-391.

56. Hibi T, Kosugi S, Iwai T, Kawata M, Seo S, Mitsuhara I, Ohashi Y: Involvement of EIN3 homologues in basic PR gene expression and flower development in tobacco plants. J Exp Bot 2007, 58(13):3671-3678.

57. Shin R, Burch AY, Huppert KA, Tiwari SB, Murphy AS, Guilfoyle TJ, Schachtman DP: The Arabidopsis transcription factor MYB77 modulates auxin signal transduction. Plant Cell Online 2007, 19(8):2440-2453.

58. Fu J, Wang S: Insights into auxin signaling in plant-pathogen interactions. Front Plant Sci 2011, 2:74

59. Koo SC, Choi MS, Chun HJ, Shin DB, Park BS, Kim YH, Park H, Seo HS, Song JT, Kang KY, Yun D, Chung WS, Cho MJ, Kim MC: The calmodulin-binding transcription factor OsCBT suppresses defense responses to pathogens in rice. Mol Cells 2009, 27(5):563-570.

60. Eulgem T, Rushton PJ, Robatzek S, Somssich IE: The WRKY superfamily of plant transcription factors. Trends Plant Sci 2000, 5(5):199-206.

61. Kim J, Yi H, Choi G, Shin B, Song P, Choi G: Functional characterization of phytochrome interacting factor 3 in phytochrome-mediated light signal transduction. Plant Cell 2003, 15(10):2399-2407. 
62. Sardanelli S, Kenworthy WJ: Soil moisture control and direct seeding for bioassay of Heterodera glycines on soybean. J Nematol 1997 29(4S):625-634.

63. Bybd DW, Kirkpatrick T, Barker KR: An improved technique for clearing and staining plant tissues for detection of nematodes. J Nematol 1983, 15:142-143.

64. Mujer C, Andrews DL, Manhart J, Pierce S, Rumpho M: Chloroplast genes are expressed during intracellular symbiotic association of Vaucheria litorea plastids with the sea slug Elysia chlorotica. Proc Nat Acad Sci 1996, 93(22):12333-12338.

65. Peterson JD, Umayam LA, Dickinson TM, Hickey EK, White O: The comprehensive microbial resource. Nucleic Acids Res 2001, 29:123-125.

66. Altschul S, Madden T, Schäffer A, Zhang J, Zhang Z, Miller W, Lipman D: Gapped Blast and PsiBlast: a new generation of protein database search programs. Nucleic Acids Res 1997, 25(17):3389-3402.

67. The UniProt Consortium: Reorganizing the protein space at the Universal Protein Resource (UniProt). Nucleic Acids Res 2012, 40(D1):D71-D75.

doi:10.1186/s12870-014-0300-9

Cite this article as: Hosseini and Matthews: Regulatory interplay between soybean root and soybean cyst nematode during a resistant and susceptible reaction. BMC Plant Biology 2014 14:300.

Submit your next manuscript to BioMed Central and take full advantage of:

- Convenient online submission

- Thorough peer review

- No space constraints or color figure charges

- Immediate publication on acceptance

- Inclusion in PubMed, CAS, Scopus and Google Scholar

- Research which is freely available for redistribution

Submit your manuscript at www.biomedcentral.com/submit

\section{() Biomed Central}

